\title{
Effect of saliva and serum on the adherence of Candida species to chlorhexidine-treated denture acrylic
}

\author{
J. MCCOURTIE, T. W. MACFARLANE and L. P. SAMARANAYAKE
}

\section{Oral Microbiology Unit, Department of Oral Medicine and Pathology, University of Glasgow Dental Hospital and School, 378 Sauchiehall Street, Glasgow G2 3JZ}

\begin{abstract}
Summary. The effect of saliva and serum on the adherence of five strains of Candida albicans and one each of $C$. tropicalis and C. glabrata to chlorhexidine-pretreated acrylic was measured in vitro. A four-fold dilution of saliva or serum significantly inactivated the fungicidal effect of chlorhexidine gluconate. Pretreatment of the acrylic with unstimulated mixed saliva for $30 \mathrm{~min}$ led to a reduced adherence for all the Candida strains tested, whilst a similar pretreatment with serum slightly increased adhesion. Moreover treatment of saliva- or serum-coated acrylic with chlorhexidine gluconate $2 \%$ reduced adherence by between $19 \%$ and $86 \%$. The inhibition of yeast adherence by chlorhexidine persisted for up to 19 days after the exposure of the acrylic strips to the disinfectant.
\end{abstract}

\section{Introduction}

In chronic atrophic candidosis (CAC), the most prevalent form of oral candidosis (Odds, 1979), yeasts are isolated more often from the fitting surface of the denture than from the palate. The ability of Candida species, especially $C$. albicans, to adhere to and colonise denture acrylic is thought to be important in the pathogenesis of this disease. Different species of Candida vary in their ability to adhere to denture acrylic in vitro and adherence is significantly reduced by pretreatment of the acrylic with chlorhexidine gluconate $2 \%$ for $30 \mathrm{~min}$; this regimen was fungicidal for all the strains tested (McCourtie et al., 1985). Serum inactivates the bactericidal action of chlorhexidine in vitro (Roberts and Addy, 1981) and it seemed likely that saliva had a similar effect. Therefore it was decided to investigate the effect of chlorhexidine on yeast growth and adherence in the presence of saliva and serum.

\section{Materials and methods}

\section{Organisms}

Five strains of $C$. albicans, and one each of C. tropicalis and C. glabrata were used. C. albicans MRL 3153 was from the Mycological Reference Laboratory, Central Public Health Laboratories, Colindale, London. C. albicans GRI 682 was isolated from a routine cervical smear

Received 13 Feb. 1985; revised version accepted 20 Mar. 1985. from an apparently asymptomatic woman and C. albicans strains GDH 2346 and GDH 2023 were from patients with CAC. C. albicans GDH 3212 was from saliva from an asymptomatic child, while $C$. tropicalis GDH 1160 and $C$. glabrata GDH 820 were isolated from a patient with CAC and from a tongue lesion respectively. Strains of $C$. albicans isolated from infections were designated I strains and those from symptomiess carriers were referred to as $C$ strains, as previously described (McCourtie and Douglas, 1984). All isolates were identified by tests for germ-tube production, sugar-assimilation and fermentation patterns (Lodder, 1970). The organisms were maintained on slopes of Sabouraud Dextrose Agar (Difco) and subcultured monthly. Every 2 months the cultures were replaced by new ones freshly grown from freeze-dried stock.

\section{Growth conditions}

The yeasts were grown in Yeast Nitrogen Base Medium (YNB: Difco) containing $500 \mathrm{~mm}$ sucrose as previously described (McCourtie and Douglas, 1981). They were harvested after $24 \mathrm{~h}$ (stationary growth phase) and washed twice in $0 \cdot 15 \mathrm{~m}$ phosphate-buffered saline $(p \mathrm{H} 7 \cdot 2$; PBS).

\section{Preparation of acrylic strips}

Acrylic strips were prepared as previously described (Samaranayake and MacFarlane, 1980) with the following modifications. Acrylic monomer and polymer (Simplex Rapid; Howmedica International Ltd, 622 Western Avenue, London) were mixed on aluminium foil-covered glass slides and polymerised in a water bath at $40^{\circ} \mathrm{C}$. The 
aluminium foil aids recovery of the acrylic from between the slides and the lower temperature for polymerisation reduces bubbling within the acrylic and increases clarity.

\section{Adherence assay}

Pools of unstimulated mixed saliva and of serum from several donors were prepared. The saliva was clarified by centrifugation at $17000 \mathrm{~g}$ for $30 \mathrm{~min}$ at $4^{\circ} \mathrm{C}$. To observe the effect of saliva and serum on yeast adherence, acrylic strips were preincubated in clarified saliva or serum for 30 min at room temperature; control strips were similarly preincubated in PBS. Some acrylic strips were further treated with chlorhexidine gluconate (ICI Pharmaceuticals Ltd, Macclesfield) $2 \%$ or with sterile distilled water at room temperature for $30 \mathrm{~min}$ before performing the adherence assay.

To determine how long acrylic-bound chlorhexidine inhibited yeast adherence, acrylic strips were incubated in chlorhexidine gluconate $2 \%$ at room temperature for 30 min and then stored in a moist chamber or submerged in filter-sterilised (Sterifil-D filter unit, Millipore Corporation) clarified saliva until required for adherence assays. Control strips were similarly pretreated with distilled water and stored in the same way. The adherence assay was as previously described (McCourtie and Douglas, 1981). Briefly, transparent acrylic strips were incubated in standardised yeast suspensions $\left(2.5 \times 10^{9}\right.$ cells $/ \mathrm{ml}$ in PBS), and the number of adherent organisms determined by microscopy. All adherence values quoted were obtained by counting 30 fields on each of duplicate strips from 10 independent assays.

\section{Sensitivity to chlorhexidine gluconate}

Fungicidal concentrations of chlorhexidine for the yeast isolates were determined as previously described (McCourtie et al., 1985). To examine the effect of saliva and serum on the fungicidal concentrations, standardised yeast suspensions $\left(5 \times 10^{9} \mathrm{cells} / \mathrm{ml}\right.$ in distilled water) were added to $1-\mathrm{ml}$ volumes of various concentrations of chlorhexidine containing saliva or serum at dilutions of 1 in 4,10 and 20 to yield final yeast concentrations of $2.5 \times 10^{9}$ cells $/ \mathrm{ml}$.

\section{Statistical analysis}

Student's $t$ test was used to evaluate differences in yeast adherence. A p value of $<0.05$ was considered significant.

\section{Results}

Effect of saliva and serum on the sensitivity of Candida species to chlorhexidine

The fungicidal concentrations of chlorhexidine gluconate with various concentrations of serum and saliva for the Candida species tested are shown in the table. When the assay mixture contained either serum or saliva diluted 1 in 4 there was a marked increase in the fungicidal concentration of chlorhexidine for all the isolates; up to ten times more chlorhexidine was required to achieve the same effect as in the control assays. However, further dilution of serum or saliva reduced its effect and the fungicidal concentrations of chlorhexidine returned to those of control assays when either serum or saliva was diluted 20 -fold.

\section{Effect of saliva and serum on yeast adherence}

Adherence of all Candida strains was reduced by pretreatment of the acrylic with pooled unstimulated mixed saliva for $30 \mathrm{~min}$ at room temperature

Table. Effect of saliva and serum on the sensitivity of Candida species to chlorhexidine gluconate

\begin{tabular}{l|ccccccc}
\hline & \multicolumn{6}{c}{$\begin{array}{c}\text { Fungicidal concentration* of } \\
\text { chlorhexidine }(\% \mathrm{v} / \mathrm{v}) \text { in }\end{array}$} \\
\cline { 2 - 8 } & \multicolumn{6}{c}{ Saliva dilution } & Serum dilution \\
\cline { 2 - 8 } Isolate & Control & 4 & 10 & 20 & 4 & 10 & 20 \\
\hline C. albicans GDH 2023 & 0.05 & 0.2 & 0.05 & 0.05 & 0.2 & 0.2 & 0.07 \\
C. albicans GDH 2346 & 0.05 & 0.1 & 0.07 & 0.05 & 0.1 & 0.05 & 0.05 \\
C. albicans MRL 3153 & 0.05 & 0.1 & 0.07 & 0.05 & 0.07 & 0.05 & 0.05 \\
C. albicans GRI 682 & 0.1 & 1.0 & 0.2 & 0.2 & 1.0 & 1.0 & 0.5 \\
C. albicans GDH 3212 & 1.0 & 2.0 & 1.0 & 1.0 & 1.0 & 1.0 & 1.0 \\
C. tropicalis GDH 1160 & 0.02 & 0.05 & 0.05 & 0.02 & 0.05 & 0.05 & 0.02 \\
C. glabrata GDH 820 & 0.07 & 0.1 & 0.1 & 0.07 & 0.1 & 0.07 & 0.07 \\
\hline
\end{tabular}

* Organisms were exposed to chlorhexidine for $5 \mathrm{~min}$, and the fungicidal concentration taken as percentage of chlorhexidine in the lowest dilution required to achieve a $100 \%$ kill. 


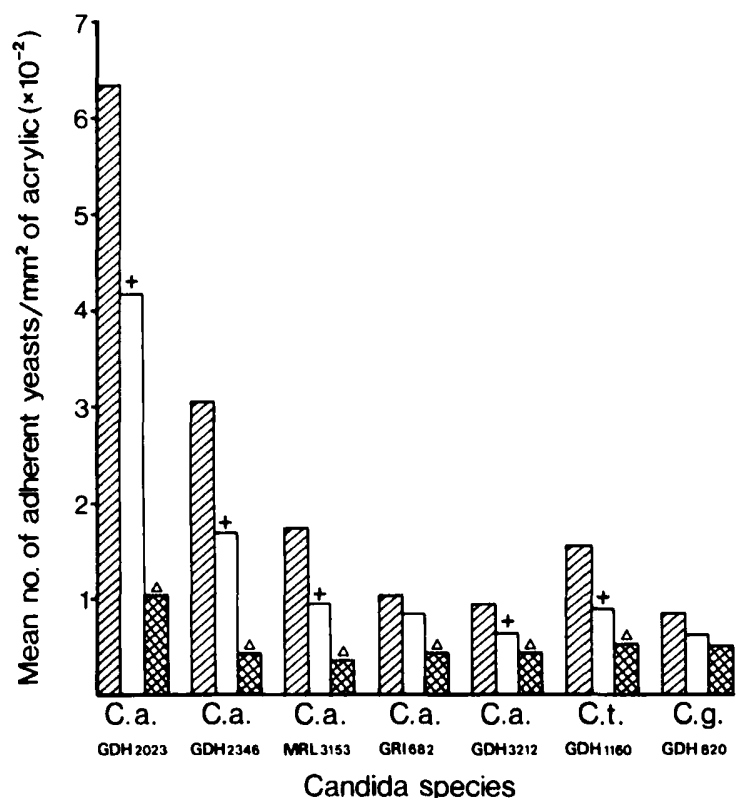

Fig. 1. Adherence of Candida strains to acrylic pretreated with distilled water ( $\square$ ), saliva ( $\square$ ), or saliva followed by chlorhexidine gluconate $2 \%$ (E). Standard errors were in the range $4.6 \%$ to $15.5 \%$ of the mean values. A significant decrease in adherence with saliva treatment of acrylic is shown $(+)$ while $(\Delta)$ represents significant inhibition of adherence to saliva-coated acrylic by chlorhexidine gluconate $2 \%$. C.a. $=C$. albicans; C.t. $=C$. tropicalis; and $\mathrm{C} .(\mathrm{g}) .=\mathrm{C}$. glabrata.

(fig. 1). Inhibition of adherence was greater with those strains that adhered to acrylic in greater numbers, i.e., the strains of $C$. albicans isolated from infections (I strain; $34-50 \%$ ) than with the strains of $C$. albicans from symptomless carriers (C strains) and with $C$. tropicalis GDH 1160 and $C$. glabrata GDH $280(15-43 \%)$. However, inhibition of adherence was not significant for all the other Candida isolates (fig. 1). Treatment of the saliva-coated acrylic with chlorhexidine $2 \%$ again reduced adherence. Once more inhibition was greatest with the I strains of $C$. albicans $(63-75 \%)$ than with the $C$ strains of $C$. albicans and with $C$. tropicalis GDH 1160 and C. glabrata GDH 820 (19-40\%).

Pretreatment of denture acrylic with pooled human serum increased adherence of all Candida strains except $C$. albicans GDH 3212 with which adherence was slightly but not significantly reduced (fig. 2). Further treatment of serum-coated acrylic with chlorhexidine $2 \%$ inhibited subsequent adherence of all strains of Candida. Again, inhibition of adherence to serum-coated acrylic was greatest with I strains of $C$. albicans $(83-86 \%)$. The significance levels of changes in adherence are given in the caption for fig. 2.

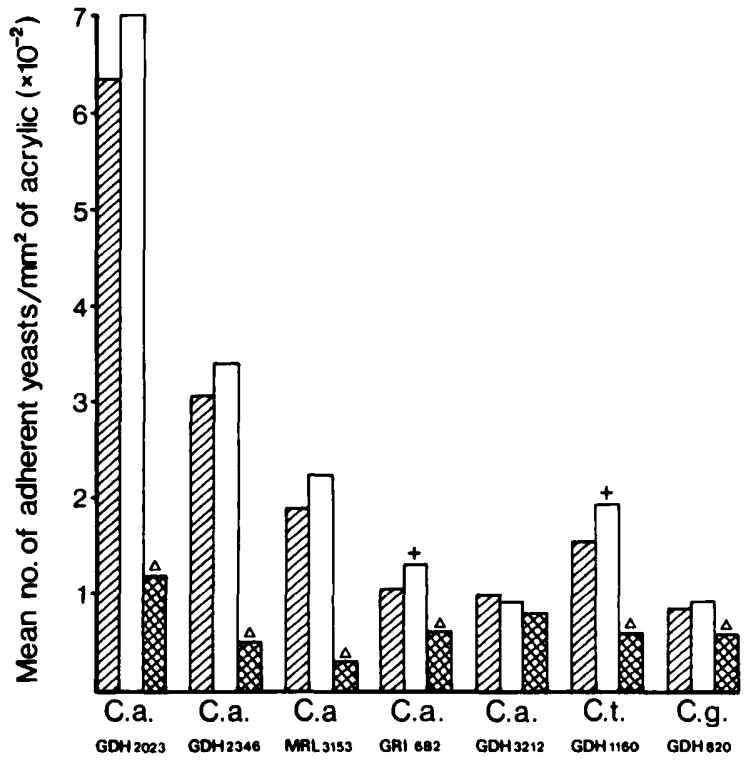

Candida species

Fig. 2. Adherence of Candida strains to acrylic pretreated with distilled water ( $\square$ ), saliva ( $\square$ ), or serum followed by chlorhexidine gluconate $2 \%$ (⿴囗大) . Standard errors were in the range 4.4 $13.1 \%$ of the mean values. A significant increase in adherence with serum treatment of acrylic is shown $(+)$ while $(\Delta)$ represents a significant inhibition of adherence to serum-coated acrylic by chlorhexidine gluconate $2 \%$. C.a. $=C$. albicans; C.t. $=C$. tropicalis; and C. $(\mathrm{g}) .=$ C. glabrata.

\section{Retention of chlorhexidine by acrylic}

Adherence of $C$. albicans GDH 2346 to denture acrylic was inhibited by chlorhexidine gluconate for up to 19 days after treatment with the disinfectant when the acrylic strips were stored in a moist chamber (fig. 3). After this time, the ability of chlorhexidine to reduce yeast adherence was rapidly lost. In an attempt to mimic the conditions in the mouth, the acrylic strips were also stored in filtersterilised mixed saliva at $37^{\circ} \mathrm{C}$. As can be seen from fig. 3, chlorhexidine-treated acrylic incubated in filtered saliva was not as efficient at inhibiting subsequent adherence of $C$. albicans GDH 2346. However inhibition of adherence of $50-60 \%$ was maintained for 19 days after initial treatment of the acrylic with chlorhexidine $2 \%$.

\section{Discussion}

Trauma to the palatal mucosa is an important aetiological factor in CAC (Nyquist, 1952; Ritchie et al., 1969) and it is likely that any inflammatory serous exudate would coat the fitting surface of the acrylic dentures. Our previous work (Samaranayake et al., 1980; McCourtie and Douglas, 1981) 


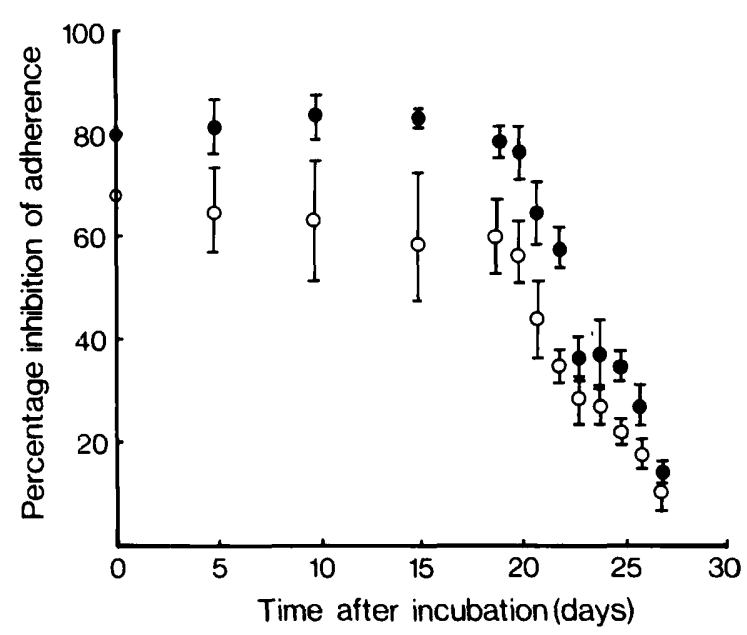

Fig. 3. Effect of time after pretreatment with chlorhexidine gluconate $2 \%$ on the subsequent adherence of $C$. albicans GDH 2346 to acrylic stored in a moist chamber $(\bullet)$ or in filter-sterilised mixed pooled saliva (O). Bars represent + and -1 SEM.

showed that adherence of $C$. albicans to serumcoated acrylic strips was enhanced, while that to saliva-coated acrylic was inhibited when compared with serum- or saliva-free control strips. The results reported here confirm these results with a range of Candida species. Furthermore, when the Candida species were treated with saliva by suspending the organisms in saliva instead of PBS, adherence to acrylic was significantly lower than to untreated acrylic (data not shown), as previously shown with C. albicans (McCourtie and Douglas, 1981). The attachment of certain oral bacteria to hydroxyapatite is similarly inhibited by saliva (Clark and Gibbons, 1977; Gibbons and van Houte, 1980). Our results also support the in-situ observations of Olsen and Haanaes (1977) that yeast colonisation of acrylic plates worn by monkeys with a reduced salivary flow was increased. Furthermore, chronic oral candidal infections are commonly seen in patients with Sjögrens syndrome, whose salivary flow is absent or minimal (MacFarlane and Mason, 1974).

Roberts and Addy (1981) and Hennessey (1973) found that serum markedly reduced the bactericidal action of chlorhexidine. Although in our study serum and saliva considerably reduced the fungi-

\section{REFERENCES}

Bonesvoll P, Lokken P, Rolla G, Paus P N 1974a Retention of chlorhexidine in the human oral cavity after mouth rinses. Archives of Oral Biology 19:209-212.

Bonesvoll P, Lokken P, Rolla G 1974b Influence of concentration, time, temperature and $\mathrm{pH}$ on the retention of chlorhex- cidal activity of chlorhexidine for all isolates of Candida tested, they had little effect on the ability of chlorhexidine to inhibit adherence of Candida species to acrylic when compared with chlorhexidinemediated inhibition of adherence to untreated denture acrylic (McCourtie et al., 1985).

It is thought that chlorhexidine is effective as an oral disinfectant because it is retained on oral surfaces and then slowly released into the oral cavity (Bonesvoll et al., 1974a). After a 1-min rinse with $10 \mathrm{ml}$ of chlorhexidine $0.2 \%$, Bonesvoll et al. (1974a) found a sharp fall in the concentrations of chlorhexidine in saliva during the first few hours followed by slow release with bactericidal activity still present after $24 \mathrm{~h}$. Oral retention was approximately proportional to the concentration of chlorhexidine used in the range tested $(0.05-0.4 \%$; Bonesvoll et al., 1974b). With the higher concentrations of chlorhexidine used in this study $(2 \%)$ inhibition of adherence of $C$. albicans to denture acrylic persisted for 19 days after treatment of the acrylic. However it is likely that in vivo the constant washing action of saliva would reduce this period of activity. It is interesting to note in this context that the application of a chlorhexidine acetate-impregnated polymer film to the surface of a removable partial denture significantly reduced plaque formation in vivo for up to 12 days while in-vitro growth of Streptococcus mutans was inhibited for as long as 45 days (Hirschfeld et al., 1984). Moreover Schaeken et al. (1984) found that teeth treated for $5 \mathrm{~min}$ with chlorhexidine gluconate $5 \%$ in carboxymethylcellulose gel (the gel reduces the release rate of chlorhexidine) significantly reduced the numbers of Str. mutans for 21 days, compared with counts at untreated sites.

The results of this investigation coupled with our previous work (McCourtie et al., 1985) suggest strongly that soaking acrylic dentures with chlorhexidine gluconate $2 \%$ for $15-30$ min should be an effective measure in the treatment of CAC. The regular use of this would be useful in preventing a recurrence of $\mathrm{CAC}$ in patients susceptible to this condition.

This work was supported by a grant from ICI Pharmaceuticals Ltd, Macclesfield, England.

idine in the human oral cavity after mouth rinses. Archives of Oral Biology 19:1025-1029.

Clark W B, Gibbons R J 1977 Influence of salivary components and extracellular polysaccharide synthesis from sucrose on the attachment of Streptococcus mutans 6715 to hydroxyapatite surfaces. Infection and Immunity 18:514-523.

Gibbons R J, Van Houte J 1980 Bacterial adherence and the 
formation of dental plaques. In: Beachey E H (ed) Bacterial adherence (Receptors and recognition, series B, Vol. 6) Chapman and Hall, London. pp 61-104.

Hennessey T S 1973 Some antibacterial properties of chlorhexidine. Journal of Periodontal Research, 8 Suppl 12:61-67.

Hirschfeld Z, Friedman M, Golomb G, Ben-Yaacov D 1984 New sustained release dosage form of chlorhexidine for dental use: Use for plaque control in partial denture wearers. Journal of Oral Rehabilitation 11:477-482.

Lodder A 1970 The yeasts, a taxonomic study, 2nd edn. North Holland, Amsterdam.

MacFarlane T W, Mason D K 1974 Changes in the oral flora in Sjögren's syndrome. Journal of Clinical Pathology 27:416419.

McCourtie J, Douglas L J 198 I Relationship between cell surface composition of Candida albicans and adherence to acrylic after growth on different carbon sources. Infection and Immunity 32:1234-1241.

McCourtie J, Douglas L J 1984 Relationship between cell surface composition, adherence and virulence of Candida albicans. Infection and Immunity 45:6-12.

McCourtie J, MacFarlane T W, Samaranayake L P 1985 Effect of chlorhexidine gluconate on the adherence of Candida species to denture acrylic. Journal of Medical Microbiology 20:97-104.

Nyquist G 1952 Study of denture sore mouth: investigation of traumatic, allergic and toxic lesions of oral mucosa arising from use of full dentures. Acta Odontologica Scandinavica 10, Suppl 9.

Odds F C 1979 Candida and candidosis. Leicester University Press, Leicester.

Olsen I, Haanaes H R 1977 Experimental palatal candidosis and saliva flow in monkeys. Scandinavian Journal of Dental Research 85:135-141.

Ritchie G M, Fletcher A M, Main D M G, Prophet A S 1969 The aetiology, exfoliative cytology and treatment of denture stomatitis. Journal of Prosthetic Dentistry 22:185-200.

Roberts W R, Addy M 1981 Comparison of the in vivo and in vitro antibacterial properties of antiseptic mouthrinses containing chlorhexidine, alexidine, cetyl pyridinium chloride and hexetidine: relevance to mode of action. Journal of Clinical Periodontology 8:295-310.

Samaranayake L P, MacFarlane T W 1980 An in vitro study of the adherence of Candida albicans to acrylic surfaces. Archives of Oral Biology 25:603-609.

Samaranayake L P, McCourtie J, MacFarlane T W 1980 Factors affecting the in vitro adherence of Candida albicans to acrylic surfaces. Archives of Oral Biology 25:611-615.

Schaeken M J M, De Jong M H, Franken H C M, Van der Hoeven J S 1984 Effect of chlorhexidine and iodine on the composition of the human dental plaque flora. Caries Research 18:401-407. 